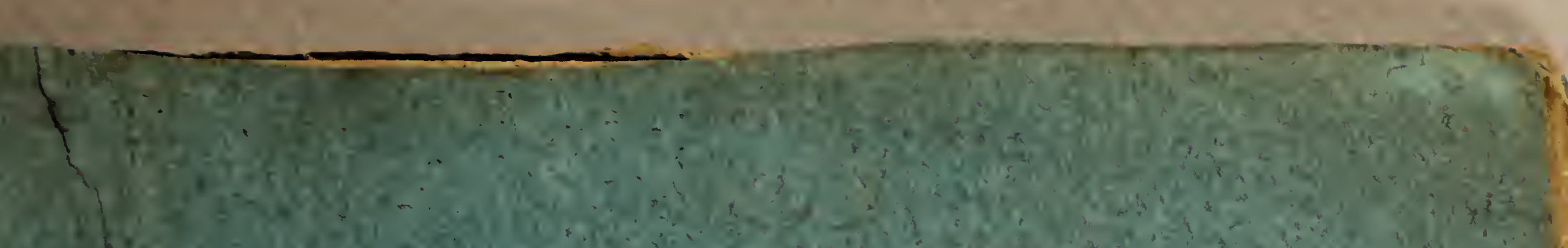

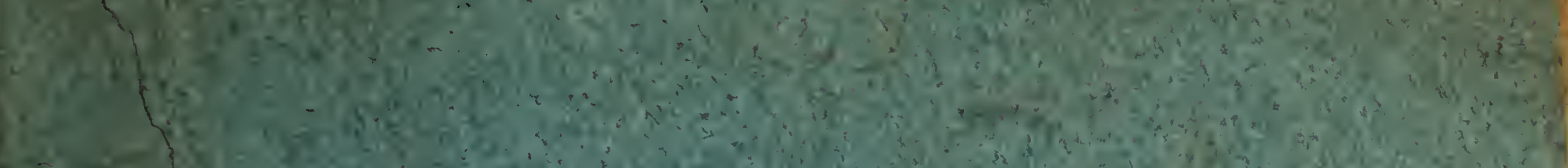

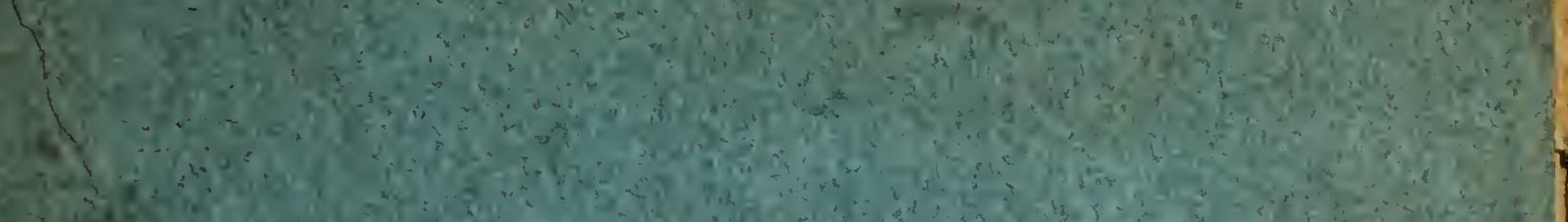

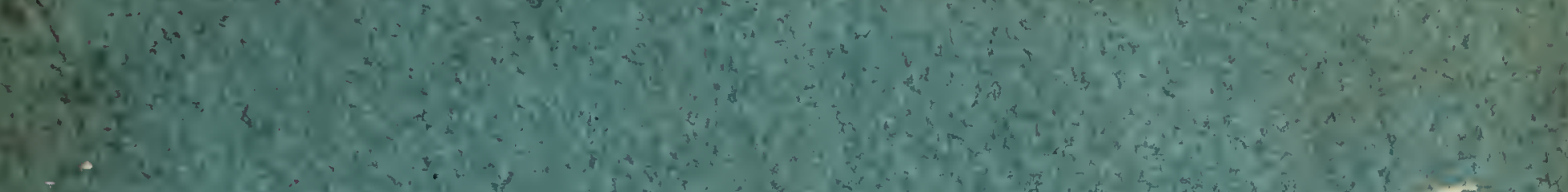

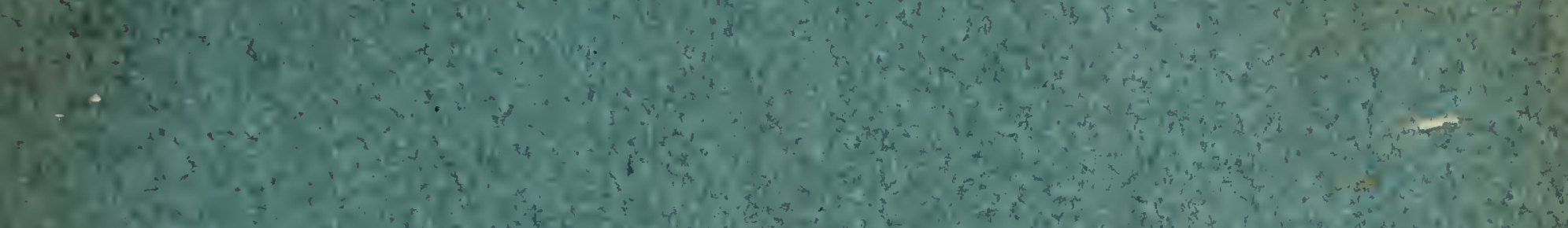

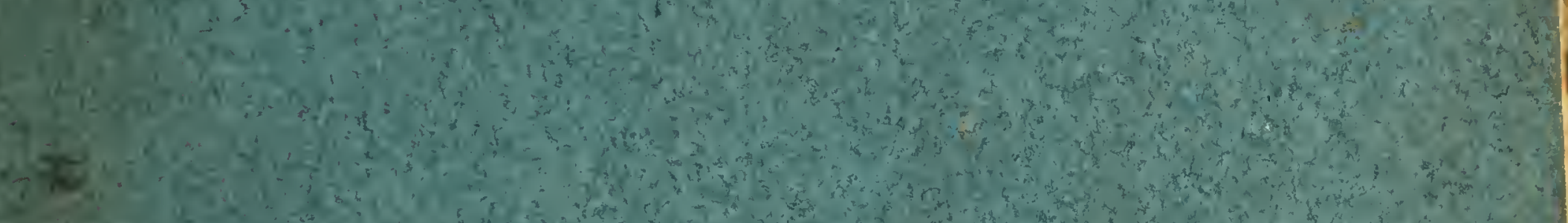
128.

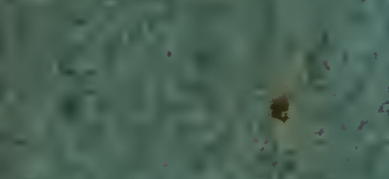

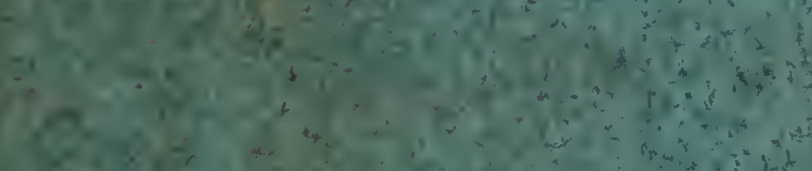
(196)

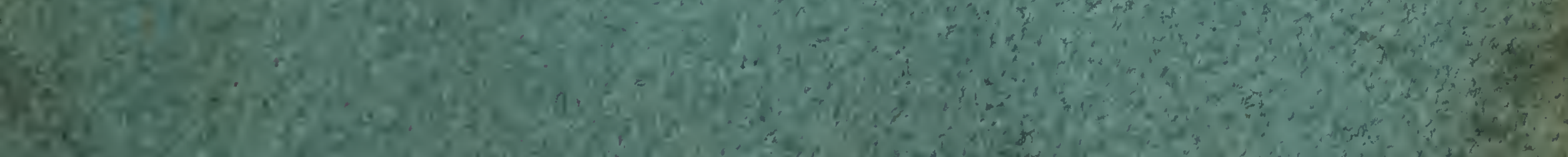

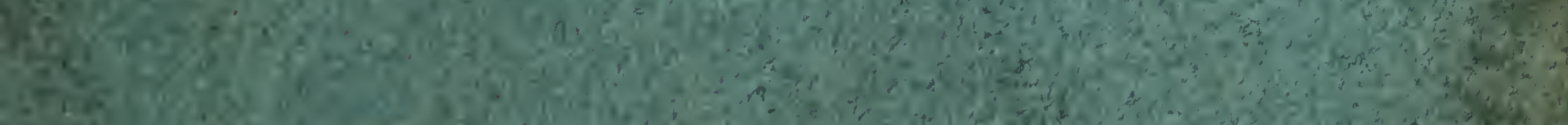

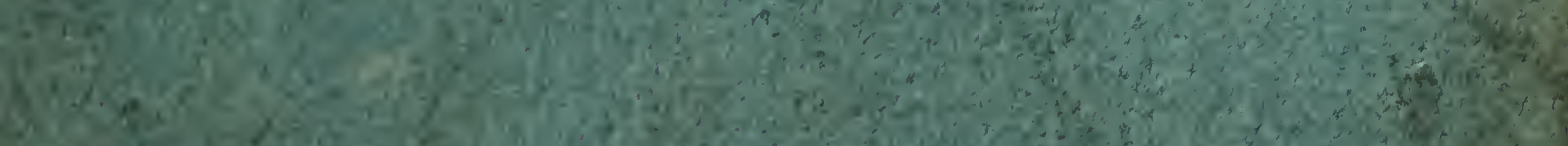

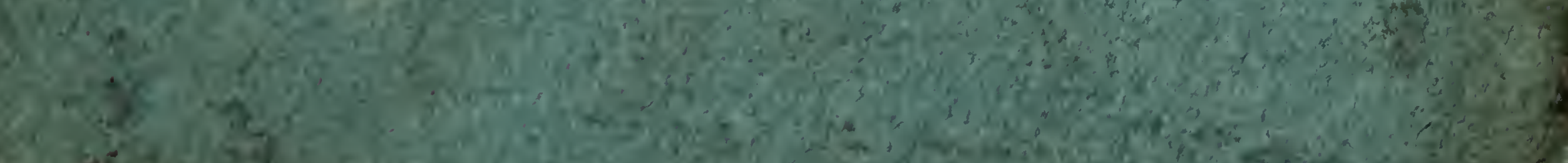
ate 


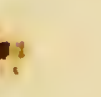

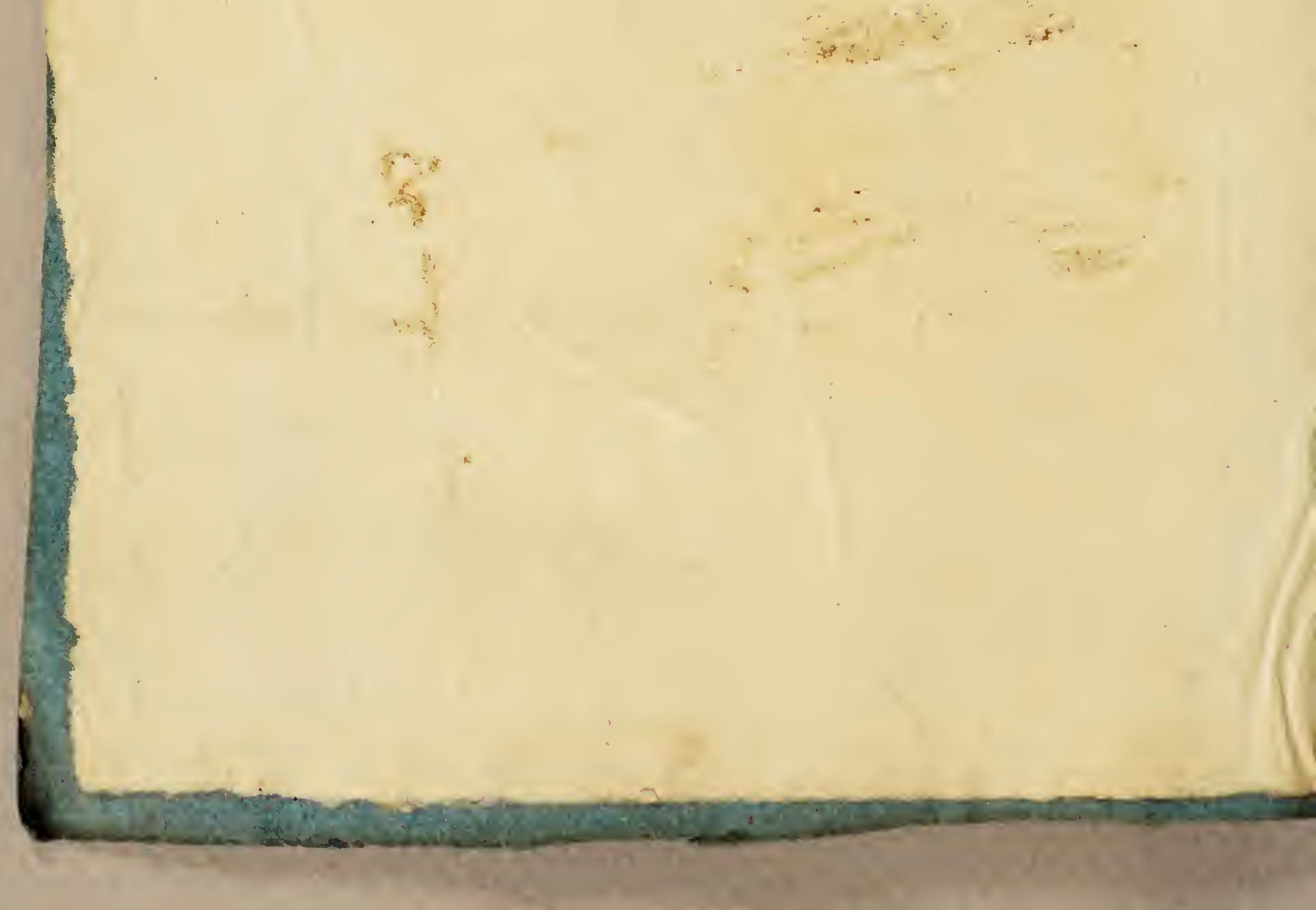


SUR LA DÉCOCTION DE T A B A C

EMPLOYÉE AU TRAITEMENT ID IE TEA G GAL TLE,

O U

Méthode de guérir cette maladie sans onguent,

Par F. J. BÉCU, Médecin des Hópitaux militaires.

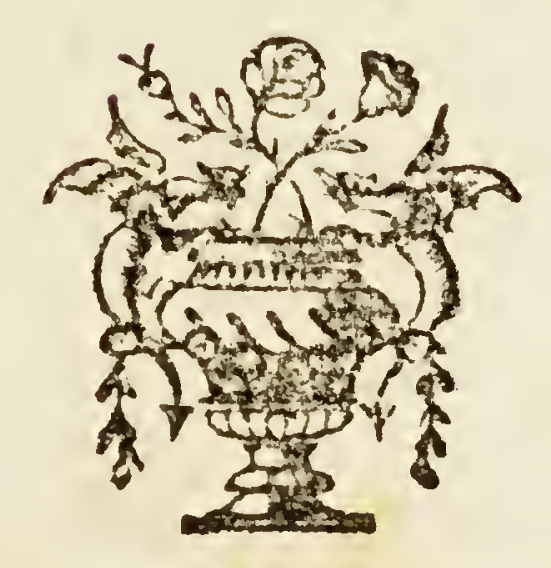

$$
\text { A L I LLE, }
$$

De l'mprimerie de C. L. DEBOUBERS, Place de Rihour.

1793. 


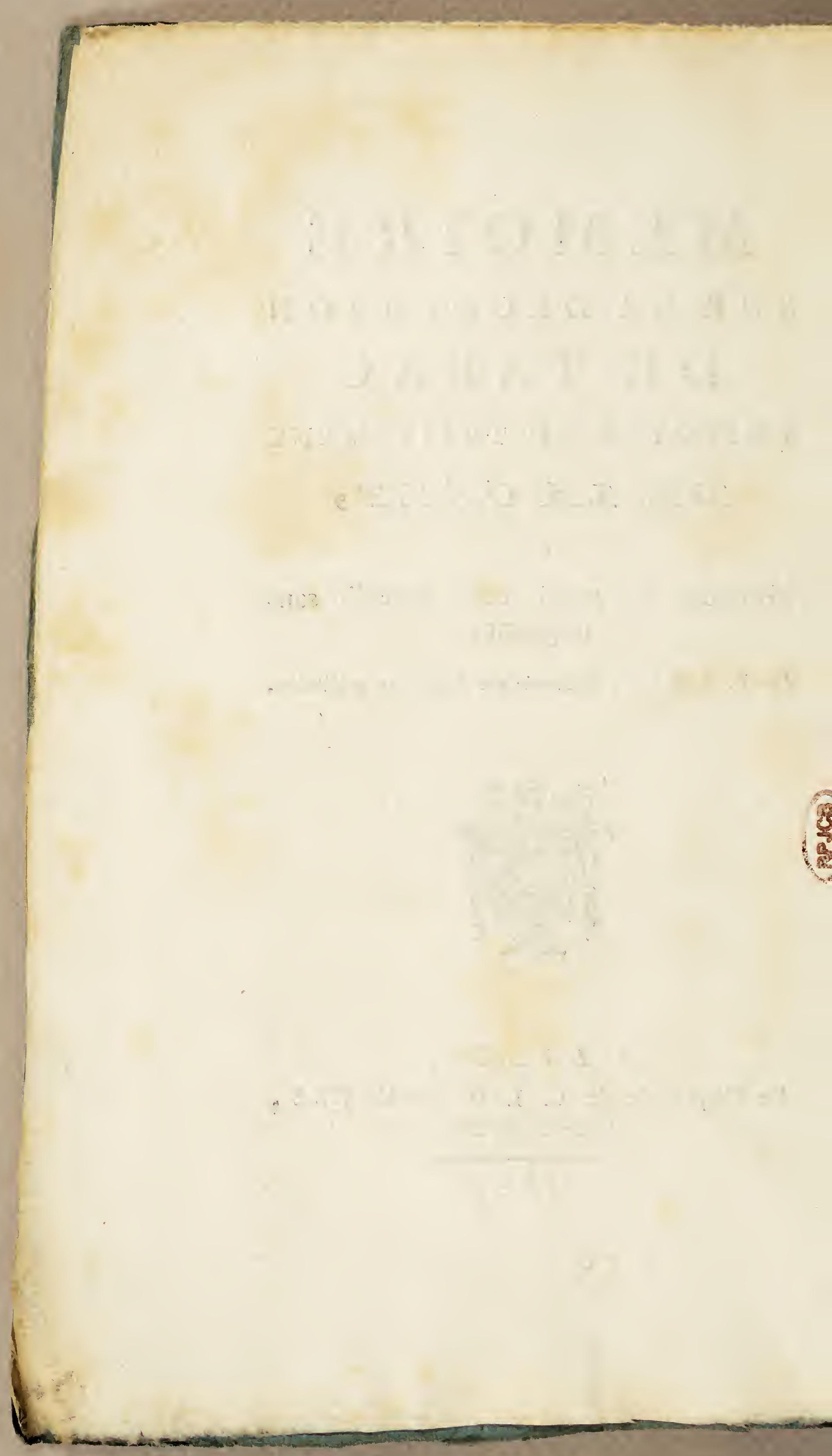




\section{SUR LA DECOCTION}

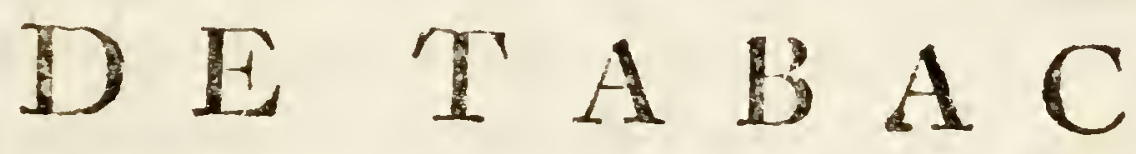

Employée au Traitement de la Gale.

\section{M}

1. 1 a dessein n'est pas de présenter un 'Traité sur la Gale : il n'entre dans mon plan, ni d'analyser la nature de cette affection cutanée, ni de discuter les principes qui la constituent \& la rendent le plus souvent contagieuse. Est-elle dae à un levain particulier, ou est-elle occasionnée par des animalcules, comme l'ont pensé Rédi, Méad, Dessault \& d'autres célèbres mélecins? Ces questions curieuses appartiennent à l'histoire naturelle \&x it la théorie de la mécecine; mais, malgré les expériences tmninenses d'un habile naturaliste de nos jours, le citoyen Olivier, elles n’ont pas encore franchi le dédale des conjectures. Un objet plus intéressant pour l'humanité a pomx le bien de la république, c'est la recllerche des moyens de' guérir cette maladie promptement \& sans danger. La nature les offre en foule, \& l'art a su les employer avec succès. Mais si, au milieu de cette moltitude de sccours, il en étoit un rui, réunissant les 


\section{(4)}

gtalités arantagouses des autres, fut exempt des inconłéniens que l'expérience journadière fat reconnotre dans ceur-ci; s’il joignoit à la promptinde \& à la sûreté de la guérison plus de simplicité dans la méthode, plus de propreté dans livsage, \& the diminution considérable dans lá dénense, sans donte coluith mériteroit la préférence par-toat \& sur-tont dans les hopitanx militaires. Mais nvant d'établir les motifs de cette préférence, il est bon de jotter un coup-d'ail précis \& critique sur la phupart des remèdes employés contre la gale.

On sait gue les préparations de sourre, de mercure \& de plomb, mêlées avec des huiles ou d'autres substances grasses, forment les différens onguens antipsoriques. Un mêlange de fleurs de soufre, de sel ammoniac \& d’axonge; l'onguent napolitain ; longuent mecuriel.citrin; lapommade de Rivière, faite avec le mercure précipté blanc \& l'onguent rosat; celle de Gardane, composée de subimé corrosif, de céruse \& de beurre frais; longnent de Van-swieten ou l'xutions minéral lié avec la graisse; tels soni les principaux bpiques employé; an traitement de la gale: la poudre antipsorique de Leclercq, dont on s'est servi dans quelques hopitanx militrires, en la délayant avec de lhuile, est encore un composé de quelques substances äres \& dessicatives dont le sonfre forme la hase; on a heau vouloir le déguiser, son odenr le décèle toujours.

Outre ces onguens a base minérale, on en 


\section{(5)}

compose encore avec les corps gras \& les sucs ou extraits de mlusieurs végétanx : tels sontla Scille \& liellebore, dont les bergers se servoient an rappost de Virgile (A) telle est la Passerage ( Lepidium latifolimn Lin.) adoncie avec la cire \& l'onguent rosat, vantée par Pline $(B)$ comme un remèce capable d'enlever facilement jusruaux moindres traces de la gale \& de la lepre. Telle est la décoction de tabac dans thuile on l'uxonge déjà indignée par Dodnens, (C) Gaspard Bauhin \&o Mathiole, (D) dont la formule a été copiée dans plusieurs pharmacopées, mais dont le citoyen Souville a donné une préparation plus exacte; (L) telle est la dentelaire annoncée par le ci. toyen Sumeire (F) \& gni a justifé la promesse de son autenr: telles sont les racines de parelle, d'hellebore blane, de chéidoine, de scrophulaire, d'angélique, \&xc.; toutes ces substances bouillies dans lituile, te heurre ou la graisse, ont fourni autant de remòdes recommandés contre la gale.

Les galenx, après avoir été saignés \& purgés, enduisent on frottent plus ou moins de fois les parties couvertes de boutons avec quelqu'une de ces préparations; le terme
(A) Geor. lib. III.
(B) Histor. nat. Lib. XX. cap. 17.
(C) Dod. stir. hist. pempt. 3.a lib. 4, pag. 456.
(D) Comment. in lib. 14 dinscor.
(E) Iournal de méd. milit. janvier 3786.
F), Ibidem tom I, pag. $1,3$.

A 2 
moyen de la durée du traitement, lorsque la gale n'est ni invétérée ni compliquée, est ordinairement de 15 a 16 jours dans les hôpitaux; \& si le traitement domestique $\&$ isolé est moins long, les raisons de cette différence sont palpables.

Il seroit inutile de rappeler ici un plus grand nombre de reccttes antipsoriines. Il n'est pas de bonne femme ni d'empiripue qui ne se dise ell possession de guelqne secret pour guérir en peu de tems tonte some de gale. Les médecins n'en ont qu'un, mais il est sûr ; c'est de traiter cette maladie méthodiquement, avec comnoissance de cause \& en distinguant ses espèces: ils savent que les topiques n'y suffisent point toujours; qu'il se rencontre des circonstances où leur action doit être précédée ou secondée de l'usage approprié des remèdes internes. C'est le cas des gales ou compliquées ou invétérées; ils n'ignorent pas qu'il en est de spontanées, qu'il en est même de critiques, où le moyen le plus assuré de les faire cisparoître promptement, deviendroit en mêneterns la source de métastases \& de répercussions funestes; toutes ces gales font exception; leur tráitement est subordonné aux circonstances qui les accompagnent, \& c'est une vérité pratique trop reconnue des médecins, pour qu'il soit nécessaire d'y insister davantage. Il ne peut donc être question ici que des gales simples ou de celles qui auroient été amenées par la sagesse du médecin au point de ponvoir être trairées comme telles ans danger. Après cette prof́ession de foi, 
que je me dispenserai de répéter, la méthode que je propose, ne pent essuyer d'objections; mais j'en ai plusieurs à opposer aux procédés dont on se sert le plus communément.

Les différens topiques graissenx dont nous venons de faire mention, appliqués d'une manière convenable $\&$ avec les précautions requises, sont sans contredit des moyens propres à guérir la gale; l'expérience le démontre tous les jours: mais ne sont-ils sujets ¿̀ aucun inconvénient? ne donnent-ils pas trop souvent naissance à des maladies plus graves que celle quilis font disparoitre? Les onguens, en général, en bouchant les pores de la peau, dérangent la transpiration \& occasionnent des rhumes, des fièvres, des maux de gorge, des cliarrlées, des érésipèles, des dartres, \&c. Une infnité d'exenples prouve qu'en dissipant la gale, au lieu d'en détruire la cause, ils n'en opèrent que la répercussion; \& l'on sait quelles penyent: être les suites de cet accilent. Le danger de la répercussion angmente, si les corps gras sont alliés à quelque préparation do plomb. Aussi Gardane, en détaillant la manière d'employer sa pommade antipsorinue, recomminde-t-it de purger it plusieurs reprises cenx qui en font usage; sans doute afin d'évacuer lhumeur galeuse par les sellos \& d'empècher qualle ne se jetre sur quelque partie essentielie à la vie.

Les ongnens sulfimreux, qui sont les plus usités, n’agissent efficacement que sur les A. 3 
gales simples \& récentes: d'ailleurs leur odeur est infecte.

Ceux qui ont le mercure pour base, excitent le plus souvent une salivation incommode \& font naître chez les sujets foibles ou cacochymes, des affections scorbutiques, des ulcères à la bonche, \&rc.

Mais un inconvénient commun à toutes ces préparations dont les substances grasses forment l'excipient, c'est la malpropreté inévitable qu'elles entrainent dans tous les lieux où un grand nombre d'hommes rassemblés sont soumis au même traitement, comme dans les hôpitaux. Les fournitures sur lesquelles sont déposées chaque jour de nouvelles couches d'onguent, de crasse \& de matière psorique, s'en imprègnent si intimement, que les lessives les plus soignées ne les en débarrasseroient quavec peine. Mais ceite difficulté même \& la crainte de contracter la gale, autorisent, en quelque sorte, des lavages si superficiels, que la différence des fournitures qui les ont éprouvés d'avec celles qui en ont besoin, est presqu'imperceptible. De cet abus, que la nature du traitement consacre, pour-ainsi-dire, en dépit des précautions les plus exactes pour le renorivellement des fournitures, s'ensuivent la détérioration \& la perte des matelas, des paillasses, des draps, des chemises, des couvertures; la contagion que répand cette insoutenable malpropreté, l'odeur méphitique qui résulte de tout ce mêlange de parties sulfurenses, mercurielles ou saturnines, alliées aux minsnes psoriques exaltés par la chateur des 


\section{(9)}

salles, toutes ces exhalaisons nesont pas noins nuisibles à la santé des malades, que désagréables pour ceux qui par état sont obligés de leur donner des soins. La réunion dune foule de galeux entassés les uns sur les autres augmente encore ce foyer d'insalubrité, sur-tout dans les tems où le froill de l'atmosphère ne permet pas de tenir les fenêtres ouvertes autant qu'il seroit convenable; il arrive souvent alors que les sujets d'une santé délicate sont obligés de quitter la salle des galeux \& de passer dans d'autres, pour y être traités de quelqu'indisposition ou inaladie qu’ils ont contractée dans la première.

Tels sont les désagrémens \& les suites fâcheuses qu'entraîne inévitablement dans les hôpitaux l'usage des antipsoriques unis aux corps gras. I'höpital militaire de Lille, oix règnent d'ailleurs l'ordre le plus exact \& la propreté la plus scrupuleuse, n'étoit pas exernpt de ces inconvéniens, \& le contraste offert par la salle des galeux comparée au reste de l'hôpital, étoit plus frappant encore, lorsqu'on y employoit le remède de Leclerca. C'est dans cette circonstance que je fus chargé en 1785 de la visite joumalière de cecte salle. Si le zìle de mon devoir diminnoit quelque chose de ma répugnance personnelle, ma sensibilité n'étoî pas moins affectée à la vue d'une quantité plus ou moins considérable d'individus livrés à toutes les horreurs de la malpropreté \& à linfluence pernicieuse d'un air nécessairement surchargé de tant de miasmes corrompus. Il'nen falint 


\section{(10)}

pas davantage pour m'engager àdes recherches dont le succès put les garantir de ces maux.

Je savois que tout l'art consiste à déterminer l'éruption galeuse à la peau par une irritation modérée, à faire suppurer les boutons autant qu'il est possible, \& enfin à les dessècher. Les topiques âcres \& les cathérétiques légers ayant tous cette propriété, je ne doutois pas que l'on put se passer des substances minérales, \& que l'un ou l'autre des onguens rapportés par Pline \& Gaspard Bauhin, ne fut très-efficace même contre les gales rebelles, invétérées \& compliquées de dartres, en y ajoutant un peu de teinture ou de poudre très-fine de cantharides. Mais il étoit principalement question d'obtenir les bons effets de ces topiques, \& d'éviter le grand inconvénient du mêlange des corps adipeux. Le règne végétal m'offroit une grande quantité de plantes propres à remplir ces indications d'une manière plus ou moins active. La dentelaire ne croissant pas dans les climats septentrionaux de la France, je jettai particulièrement mes vues sur le tabac, (nicotiana tabacum Lin.) parcè qu'il est communément cultivé dans la ci-deriant Flandre, \& que je l'avois vu employer avec succès à la campagne pour la gale des moutons \& des chiens; d'ailleurs, l'illustre Boerrhave, dans son histoire des plantes du jardin académique de Leyde, avoit fait l'éloge le moins équivoque des propriétés externes du tabac dans les affections cutanées \& psoriques : Lemery en avoit vanté l'usage, \& Dodoëns qui pra- 


\section{$(11)$}

tiquoit la médecine dans les pays - bas, l'avoit annoncé comme un moyen usité de son tems pour le traitement de ces maladies.

Appuyé de ces autorités, j'essayai avec confiance l'infusion aqueuse du tabac sur quelques pauvres infectés de la gale; je les en fis frotter, sans aucun préliminaire, aux endroits où se trouvoient les pustules galeuses : le succès surpassa mon attente. Je proposai cette méthode à mes chefs (j'étoịs alors médecin surnuméraire) \& au citoyen Coste, lors de son inspection à l'hôpital militaire de Lille. Ce médecin m'encouragea par sa propre expérience; c'étoit l'infusion de tabac dans le vin dont il avoit éprouvé les effets les plus heureux à Calais. La simpie décoction aqueuse que javois déja tentée avec avantage, nous parut préférable, parce qu'elle est moins dispendieuse. Le 13 novembre 1785 , je commençai a traiter six soldats à l'hopital militaire de Lille; je continuai ce procédé sur la moitié des galeux qui se rendoient à l'hûpital, tandis que l'autre moitié se frottoit arec l'onguent de Leclecq. Cependant la différence des deux remèdes devint un sujet de jalousie entre les galeux; ceux qu'on soumettoit au liminent, se plaignoient de n'être pas traités comme leurs camarades qu’ils voyoient guérir plus tôt qu'eux, \& d'une manière moins désagréable. Les chefs de l'hôpital mirent fin à ces débats; convaincus de la supériorité de la nouvelle méthode, ils en établirent l'usage, bannirent les onguens de la salle des galeux, \& bientôt, ̀̀ la malpropreté, à l'infection, en 
un mot à l'aspect hideux que présentoit cette salle, on vit súccéder la netteté, la salubrité \& la décence. (a)

Voici notre manière de préparer \& d'administrer le remède: on prend deux livres de feuilles séchées du meilleur tabac C. D. du plus âcre : après les avoir hâchées, on les met infuser pendant douze heures au moins dans huit pintes d'eau bouillante, mesure de Paris, ou bien on les fait bouillir légèrement dans neuf pintes du même véhicule pour les réduire à-peu-près à la première quantité : on dissout dans l'eau,

(a) Qui croiroit, ou plutòt qui ne croiroit pas que le despotisme des agens di gouvernement d'alors, s'étendit jusques sur le plus libre de tous les arts, l'art de guérir? Monseigneur l'Intendant de Flandre, qui protégeoit un nommé Leclercq, \& qui vouloit qu'on se servit exclusivement du prétendu secret de cet empirique, trouva fort mauvais qu'on eut introduit à Thôpital de Lille une nouvelle méthode de guérir la gale. J'eus l'insolence de représenter à sa Grandeur que ce moyen étoit plus économique, qu'il guérissoit plus promptement, \& qu'il n'avoit point les inconvéniens de l'onguent fétide \& malpropre qu'on employoit auparavant; que d'ailleurs il seroit aussi absurde que contraire au bien du service des hopitaux, d'entraver ainsi les officiers de santé dans le choix \& la prescription des remèdes, \&c. Cette témérité me valut de la part de Monseignenr, de petites douceurs dont je conserve encore l'agréable souvenir. Je reçus d'itératires défenses de me servir davantage du tabac, avec ordre impératif \& menaçant de reprendre le remède de Leclercq que $M$. l'intendant avoit jugé préférable à tout autre. Pendant cette lutte qui dura plusieurs mois, Leclecq mourut : cet incident \& l'intervention du ministre, mirent fin au procês; le secret admíable fut oublié avec son inventeur. 


\section{(13)}

ayant d'y mettre le tabac, me once de sol ammoniac; mats comme ce sel cet assez cher, nous lui substimons denx onces d'a! xali fixe, soit végétal, soit minéral, \& le remede n'y perd point le son efricacité. Nous nous servons ordinairement de la soude \& nous fa:sons boullir le tabac. Ces substances salines aident à en extraire les principes, clles forment une espoce de savon avec sa partie huilense \& rendent par ce moven l'action du renède plus conce, sans en diminuer l'énergie : on donne à chaque galeux, dans un petit pot de terre, cinq onces de cette décoction tiode ; il la fait chaufier, lorsqu'elle est refroilie; il en prend dans le creux de la main, ou bien il en imbibe un morcean de linge ou d'éponge, \& s'en frotte devant le feu lorsqu'il fait froid, les poigners, les jarrets \& les autres parties du corps affectées do boutons galeax. Ces frictions durent huit a dix minutes. Les premiers jours du traitement on les répète ordinairement trois fois, le matin ì jemn, vers deux hemres aprèsunidi \& le soir avant de se coucher. On recommande aux galenx de ne les faire qu'avec ménagemeit sur le ventre \& à certaines distances de l'henre des repas. Sans cette précantion, les parties âcres \& pénétrantes du tabac, pourroient inriter l'estomac au point d'excicer des nausées \& des vomissemens.

Leffet du remèce est d'échauffer la peau, d'augmenter l'émption galeuse, de faire suinter ou sumpurer les boutons, qui bientôt amès se desschent it s'eftacent. Les sujets 


\section{$(14)$}

dont le tissu de la peau est dur, épais \& serré, doivent se frotter plus vivement que ceux qui l'ont mince, délicat \& sensible; lorsque l'éruption est bien établie, que la peau cornmence à rougir, que les boutons sont enflammés, on fait des frictions plus légères \& moins fréquentes. Il est quelquefois à propos d'humecter, de détendre la peau, d'en calmer la cuisson par un bain tiède. Cette méthode convient surtout dans l'espèce de gale quion nomme gratelle, gale sèche ou canine. Il est bon aussi d'adoucir les excoriations avec le cérat de Galien ou celui de Goulard, ou encore mieux avec ure décoction émolliente quelconque.

En général ce traitement n'exige point de préparation; lorsque la gale est simple \& récente, \& que les sujets sont bien portans lailleurs, il suffit de se frotter six, huit ou dix jours de la manière prescrite, pour être parfaitement guéri. Cependant dans le cas de pléthore ou de saburre des premières voies, wne saignée, un vomitif ou un catartique ne peuvent qu'être utiles.

Il n'er est pas de même des gales entées sur un vice scropholeux, vénérien, dartreux ou scorbutique; Iorsqu'à la longue le levain psorique a infecté les humenrs, lorsqu’il est compliqué d'érésipèle, lor qu : son éruption est la crise de quelque mal ule; on conçoit quil est inaispensable alor's, pour obtenir une guérison sîre \& radicale, de faire précéder les remèdes adaptés anx circonstances \& de les contimuer pendant le 


\section{$(15)$}

cours des frictions. (Je servis inexcusable de le répéter, contre ma promesse, si ce mémoire ne devoit être lu que par des gens de l'art. )

Le premier coup d'œil ne saisit pas torjonis le caractère on la complication de la gale. St dans les cas doutenx les frictions sentes n'ont point un effet curatif, au moins elles ne sont pas nuisibles, puisqu'elles ne font que développer l'éruption, quelqu'en soit la source. Elles servent même, pourainsidire, de pierre de touche pour reconnoître si la gale tient à quelque cause particulière. In effet, lorsque les frottemens pratiqués soigneusement douze ou quinze jours au plus, nemportent point la gale, on est en droii de la soupconner entretenue par quelque vice caché ; c'est le vénérien qui est le plus familier aux soldats; alors linspection exacte des sujets, un examen sévère de leur conduite passée, enfin leur aveu changent presque tonjours les soupçons en certitude : je dis presque toujours, car je riai garde de prétendre que la gale, quoique simple, ne prisse jamais résister dans aucun cas plus d'une quinzaine aux frictions les mienxadministrées.

Détailler ici un grand nombre d'observations de guérisons opérées par notre procédé antipsorique, ce seroit grossir inutilement ce mémoire. Daprès le tableau des galenx que jai traités à l'hiôpital militaire de Lille en ${ }_{77} 76$, \& dont le nombre mon ie à plus de mille, le terme moyen de la durée du traitement s'est trouyé respectivement de quatorze jours 


\section{(16)}

lihiver, de huit l'été, \& de neuf au printems \& en automne : ainsi la duréc moyenne pour l'année a été de dix jours. En comparant cet état avec celui des galeux traités les années précédentes avec des graisses, on trouve une différence notable, car le terme moyen. étoit, ordinairement de dix - sept d̀ dix-huit jours, comme on l'a constaté par les registres aux entrées.

Depuis le mois de Février dernier, j’ai rétabli à l'hopital de Lillo le traitement par le taíac, \& le succès est toujours le même. J'cus d'abord quelques obstacles à vaincre de la part des soldats, lesquels ne vouloient pas se servir d'un remède nouveau pour eux \& clont ils redouioient les effets, parce que quelques-uns de leurs camarades s'étant frottés trop fortement sur la region épigastrique, avoient souffert des malaises \& des vomissemons; mais leur ayant bien expliyué les précautions à prendre dans lapplication du topique, îs l'employèrent avec confiance \& n'en éprouvèrent plus le moindre accident.

On consomme pour cent galeux 1. s. U. to livres de tabac à 10 soms la livre. 2000 Deux liv. \& demie de soude a 16 sous $x$ o 0 Pour les purgatifís \& pelques accessoires ...................

Le traitement de charue individu revient à............... o 70

It est donc évident que la décoction de tabac employée de la manière que nons ve- 


\section{(17)}

nons de le décrire, guérit décidément la gale; que ce procédé est plus expéditif, plus sûr, moins désagréable \& moins coûieux que les remèdes ordinaires; qu'il n'infecte point les salles; que loin de gâter les fournitures, il aide à les déterger à raison dessucs savonneux du tabac; qu'il est exempt des dangers de la répercussion \& des antresinconvéniens qu'on reproche à juste titre anx antipsoriques mêlés arec des graisses; enfir, gu'il épargne a l'état un nombre très-considérable de journées de malades, non seulement par la promptitude de ses effets, mais encore parce quil n'expose point les galeux à des accidens consécutifs. Des avantages aussi précieux, constatés par des expériences multipliées, doivent assurer ì notre méthode la préférence sur les remèdes connus jusqu'à présent \& font désirer qu'elle soit généralement adoptéc dans les hôpitaux.

On trouve une foule de végétaux qu'on pourroit peut-être substituer au tabac. La nature libérale \& sage en foumit avec tant de profusion pour guérir la gale, qu'on ne doit être embarrassé que du choix. Outre les plantes dontil a déjà été fait miention, l'autorité des anciens \& l'analogie nous fondent a regnider comme antipsoriques celles qui suivent.

s Les clématites (clematis Iin. gen. plant. 695; clematitis Toumef. 150.) Les renoncules (ranunculns lin. ibidem 606 Tounef. 149.) Les unémones (ancmone tin. gen. plant. 649, Tournef. 147.) Les tithymales (Eaphor- 


\section{$(18)$}

bia Lin. gen. 609 , tithymalus Tournef. 18.) Les scabienses ( scabiosa lin. gen. plant. 115, Tournef. 263) La vermiculaire brûlante (sedum acre Lin. spec. plant, n. ${ }^{\circ} 15$, sedum foliis suhovatis adnatosessilibus gibbis erectiu culis, alternis, cynâ trifidâ hort. cliff. ${ }^{177}$ ) Lia moxtarde sénévé (sinapis nigra Lin. spec. n. ${ }^{\mathrm{Q}}$ 4 , sinapis siliquis glabris a pice tetragonis hort. cliff. 338 ) Le poivre du Pérou (schinus molle Iin. spec n. ${ }^{\circ} 2$, schinus foliis pinnatis, foliolis serratis impari longissimo petiolo requali hort. cliff. 483.) La coloquinte (cucumis colocynthis linr spec $n .^{\circ} 1$, cucumis foliis multifidis pomis globosis glabris hort. cliff.) Le fruit du fusain (evonymus europ peus Lin. spec. n. ${ }^{\circ} 1$, evonymus floribus plerisque quadrifidis ) Du poivre de Guinée ou corail des Jardins ( capsichin annuum Lin. spec. n. $0^{\circ}$, capsicum caule herbaceo pedunculis solitariis) La racine de jyrèthre (anthemis pyrethrum Lin. spec. n. ${ }^{\circ}$ I3, anthemis caulibrs simplicibus unifloribus decumbentibus foliis pimatomultifidis.) De jonc odorant. (acorus calamus Lin. spec. n. ${ }^{\circ}$, calamus aromaticus officinarum Bauh. Pin. 134.) Les fenilles de noyer (juglans regia Lin. spec. $n \circ 1$, juglans foliolis ovalibus glabris subserratis subcequalibus) De cornouiller (cormus mas Lin. spec. n. ${ }^{\circ}$, cornus arborea nmbellis involuorum æquantibus. (De ronce (rnbus fruticosns Lin. spec. n. ${ }^{1}$, rubus foliis guimatodigitatis ternatisque, caule petiolisque aculeatis. ( De troëne (ligustrum vulgare lin. spec. no 1.) De figuier (ficus carica Lin. spec. ficus foliis palmatis.) De laurier 


\section{(19)}

(laurus nobilis Lin. spec. $\mathrm{n}^{\circ} 5$, laurus foliis renosis lanceolatis permanentibus, floribus quadaifidis dioicis. ) D'oléandre ( nerium oleandor Lin. spec. n. ${ }^{\circ}$ 1. nerium foliis linearilanceolatis ternis. ) Le curage (polygonum bydropiper Lin. spec. n. 9 , polygonum floribus hexandris semidigynis, follis lanceolatis, stipulis subnuticis.) La sabine ( juniperus sabina Lin. spec. n.० 9 , juniperus foliis oppositis erectis decurrentibus : oppositionibus pixidatis.) La rue (ruta graveolens Lin. spec. n.o 1, ruta foliis décompositis, petalis laceris, floribus lateralibus quadrifidis.) La camelée (cueorum tricoccum Lin. spec. n. ${ }^{\circ}$, chamælea tricoccos Bauh. Pin. 462.) La staphisaigre (delphinium staphisagria Lin. spec. n.o 7, delphinium nectariis diphyllis petalo brevioribus, foliis palmatis : lobis obtusis.) La cévadille ou l'orge caustique (cevadilla officin : Monardi 343 , cevadilla hispanorum Rai histior I 246 , hordeum causticum B. Pin. 23. )

Toutes ces plantes bouillies ou infusées dans l'eau, sont plus ou moins propres à combattre le vice psorique; la dernière surtout, à raison de sa grande âcreté, doí agir avec beaucoup d'énergie ; \& s'il est vrai qu'il existe des insectes dans les pustules galeuses, soit que ces animalcules soient la cause de la maladie ou que leur développement en soit un produit ordinaire, la Cévadille les détruiroit promptement, car elle jouit au suprême degré d'une vertu anivermineuse.

La gale est une maladie si fréquente, si 


\section{( 20$)$}

répantue dans les armées, que si d'un cốté les dépenses quielle occasionne \& le tems fu'elle fait perdre aux défenseurs de la patrie, méritent un regard du gouvernement, d'un autre côté le traitement de cetie contagion est un objet digne du zèle \& de lattention spéciale des officiers de sunté. En engageant mes confrères à employer la méthode que je propose, je les invite aussi à faire des expériences sur les plantes indiquées dans ce inémoire, \& sur tant dautres qui paroissent douées de la faculté de guérir la gale. Les résultats seroient sans doute satisfaisans, \& dans chaque contrée on trouveroit sous la main des remèdes antipsoriques anssi efficaces que simples, \& qui ne coûteroient que la peine de les recreillir.

Quel’afrevise malpropreté qui accompagne le traitement de la gale dans les hôpitaux, en disparoisse pour jamais; que l'on proscrive les graisses, les onguens, ou que du moins on ne se serve des emplastiques que comme de moyens auxiliaires \& dans des cas particuliers. In vain réclameroit-on l'ancien. risage; le moment est venu où, en médecine. comme en politique, les préjugés \& la routine doirent céder à l'empire dé la raison.

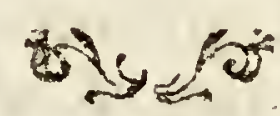


Iuh-

$$
\frac{206 / 808}{60}
$$

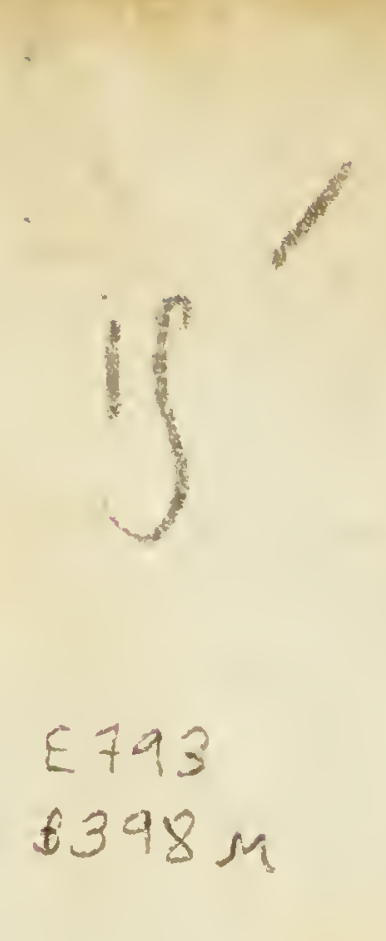


